\title{
O IMPACTO DA COVID-19 NA SUSTENTABILIDADE ECONÔMICA DAS CLÍNICAS ODONTOLÓGICAS QUE FAZEM PARTE DOS COREDES, SERRA, HORTÊNCIAS E LITORAL DO RS
}

\author{
EL IMPACTO DEL COVID-19 EN LA SOSTENIBILIDAD ECONÓMICA DE LAS \\ CLÍNICAS DENTALES QUE FORMAN PARTE DE LAS COREDES SERRA, \\ HORTEN NCIAS Y LITORAL DE RS
}

\author{
THE IMPACT OF COVID-19 ON THE ECONOMIC SUSTAINABILITY OF THE \\ DENTAL CLINICS THAT ARE PART OF THE SERRA, HORTENCIAS, AND \\ COASTAL COREDES OF RS
}

\author{
Deise Renata BRINGMANN ${ }^{1}$ \\ Maria Carolina Rosa GULLO ${ }^{2}$ \\ Thiago de Oliveira GAMBA ${ }^{3}$
}

RESUMO: Este artigo objetiva investigar o impacto da pandemia de COVID-19 no ano de 2020 nas clínicas odontológicas localizadas nos municípios que fazem parte dos Conselhos Regionais de Desenvolvimento - COREDEs, Serra, Hortênsias e Litoral do Rio Grande do Sul - RS. Neste horizonte, realizou-se uma pesquisa exploratória descritiva, que teve como instrumento para coleta de dados um questionário aplicado aos sócios-proprietários de clínicas odontológicas. Os resultados indicaram que, $61,1 \%$ das clínicas odontológicas tiveram redução no atendimento e 38,9\% não tiveram. $68,3 \%$ das clínicas tiveram redução de receita, $19 \%$ não, e $12,7 \%$ mantiveram-se economicamente estáveis. $72,8 \%$ das clínicas participantes tiveram aumento de despesas com equipamento de proteção individual. $52,4 \%$ dos respondentes têm planejamento financeiro ou uma reserva financeira para períodos de crise, enquanto 47,6 \% não possui esse recurso. Considera-se que a pandemia de COVID-19 desencadeou impactos outros que não apenas de ordem epidemiológica em escala global, repercutindo diretamente na sustentabilidade econômica das clínicas odontológicas durante o ano de 2020.

PALAVRAS-CHAVE: COVID-19. Sustentabilidade. Entidades de saúde. Impacto da pandemia.

RESUMEN: Este artículo tuvo como objetivo investigar el impacto de la pandemia de COVID-19 en el año 2020 en las clínicas dentales ubicadas en los municipios que forman parte de los Consejos Regionales de Desarrollo - COREDEs, Serra, Hortênsias y Litoral de

${ }^{1}$ Universidade de Caxias do Sul (UCS), Caxias do Sul - RS - Brasil. Doutoranda no Programa de PósGraduação em Ciências da Saúde. ORCID: https://orcid.org/0000-0002-2849-225X. E-mail:deise.renata@ucs.br ${ }^{2}$ Universidade de Caxias do Sul (UCS), Caxias do Sul - RS - Brasil. Professora Adjunta III no Departamento de Ciências Sociais. Doutorado em Economia (UFRGS). ORCID: https://orcid.org/0000-0002-3835-8222. E-mail: mcrgullo@ucs.br

${ }^{3}$ Universidade de Caxias do Sul (UCS), Caxias do Sul - RS - Brasil. Professor do Curso de Odontologia. Doutorado em Radiologia Odontológica (UNICAMP). ORCID: https://orcid.org/0000-0002-3941-9672. E-mail: togamba@ucs.br 
Rio Grande do Sul -RS. En este horizonte, se realizó una investigación exploratoria descriptiva, que tuvo como instrumento de recolección de datos un cuestionario aplicado a los socios-propietarios de clínicas dentales. Los resultados indicaron que el $61,1 \%$ de las clínicas dentales tuvieron una reducción de la asistencia y el 38,9\% no. El 68,3\% tuvo una reducción de ingresos, el 19\% no el 12,7\% se mantuvo económicamente estable. El 72,8\% de las clínicas participantes tuvieron un aumento de los gastos con equipos de protección personal. El 52,4\% de los encuestados dispone de una planificación financiera o de una reserva financiera para periodos de crisis, mientras que el 47,6\% no dispone de este recurso. Por todo lo anterior, se entiende que la pandemia de COVID-19 desencadenó impactos no sólo de orden epidemiológico a escala mundial, impactando directamente en la sostenibilidad económica de las clínicas dentales durante el año 2020.

PALABRAS CLAVE: COVID-19. Sostenibilidad. Entidades sanitarias. Impacto de la pandemia.

ABSTRACT: The objective of this article was to investigate the impact of the COVID-19 pandemic in the year 2020 on dental clinics located in the municipalities that are part of the Regional Development Councils - COREDEs, Serra, Hortênsias and the Coast of Rio Grande do Sul-RS. In this horizon, a descriptive exploratory research was carried out, which had as an instrument for data collection a questionnaire applied to the partners-owners of dental clinics. The results indicated that $61.1 \%$ of the dental clinics had a reduction in attendance, $38.9 \%$ did not. $68.3 \%$ had a reduction in revenue, $19 \%$ did not, and $12.7 \%$ remained economically stable. $72.8 \%$ of the participating clinics had an increase in expenses with personal protective equipment. 52.4\% of the respondents have financial planning or a financial reserve for crisis periods, while $47.6 \%$ do not have this resource. Given the above, it can be inferred that the pandemic of COVID-19 triggered impacts other than just epidemiological impacts on a global scale, directly impacting the economic sustainability of dental clinics during the year 2020.

KEYWORDS: COVID-19. Sustainability. Healthcare organizations. Pandemic impact.

\section{Introdução}

Este artigo objetiva investigar o impacto da pandemia de COVID-19 no ano de 2020 nas clínicas odontológicas localizadas nos municípios que fazem parte dos Conselhos Regionais de Desenvolvimento - COREDEs, Serra, Hortênsias e Litoral do Rio Grande do Sul - RS. Uma vez que as clínicas odontológicas atendem diretamente ao público, compreende-se que diante de uma crise econômica, estas podem ter adotado estratégias sustentáveis, de maneira a se manterem no mercado. Em se tratando de uma crise econômica, entende-se que os pacientes de clínicas odontológicas também podem ter se privado da atenção aos cuidados bucais, devido ao receio de contágio de Coronavírus. Essa inferência afeta diretamente a situação econômica das clínicas odontológicas, o que pode ter repercutido nos modos de conduzir a gestão da clínica. De modo a aprofundar o tema aqui apresentado, 
esta pesquisa apoia-se na literatura sobre pandemia (ATHER et al., 2020; DE OLIVEIRA; CORRÊA, 2020; MENG et al., 2020; POTT; POTT-JUNIOR, 2021; SENHORAS, 2020), sustentabilidade econômica (FERRAZ, 2020; SCHWENDICKE et al., 2020) e gestão econômica (BRASIL, 2020; DA SILVA et al., 2020; DE OLIVEIRA et al., 2020; GULLO, 2020).

Diante de um contexto pandêmico, iniciado em março de 2020 e que se estende até o período atual (maio de 2021), as clínicas odontológicas precisaram encontrar formas de buscar ou equilibrar estratégias de sustentabilidade econômica, a fim de darem continuidade à prestação de serviços que já ofereciam antes da pandemia De acordo com Gullo (2020), surgem preocupações latentes relativas ao modo de praticar uma gestão econômica com um cenário financeiro atual, de modo a não interromper suas atividades.

Esta é uma pesquisa quantitativa, que coletou 220 questionários nos municípios que fazem parte dos Conselhos Regionais de Desenvolvimento (COREDEs) ${ }^{4}-$ Serra, Hortênsias e Litoral do Rio Grande do Sul - RS. O recorte investigativo desta pesquisa deriva-se de uma pesquisa-base de doutoramento na área da Saúde, voltada à Odontologia, aprovada pela pelo Comitê de Ética em Pesquisa (CEP) da Universidade em que a pesquisa se insere (revisão às cegas), com parecer de número CAAE: 39814720.9.0000.5341. A partir dos dados da pesquisa, foi analisado sob o viés do impacto econômico quais foram as atividades eventuais ou contínuas das clínicas, que foram afetadas pela pandemia. Por meio da coleta de dados foram extraídas, para fins de sistematização da análise aqui proposta, informações que investigavam se houve aumento ou redução de gastos com recursos materiais e humanos, assim como a utilização de reservas financeiras para manter a clínica no ano de 2020. Para além disso, averiguou-se o desenvolvimento de possíveis estratégias institucionais quanto a oferta de atendimentos online, agendamentos e orientações. Portanto, esta pesquisa tem por finalidade contribuir de modo empírico para com a área da saúde, não só no que tange às consequências da COVID-19 quanto à Odontologia, mas, também com vistas a oferecer constructos teórico-metodológicos para que se possa pensar a gestão econômica de clínicas odontológicas, de modo geral.

Este artigo é constituído por Introdução; que apresenta o contexto em que se inscreve esta pesquisa, objetivo e percurso metodológico; segunda seção que trata do Covid-19 e das

${ }^{4}$ Municípios integrantes dos COREDEs que fizeram parte desta pesquisa: Caxias do Sul, Farroupilha, Bento Gonçalves, Veranópolis, Nova Prata, Carlos Barbosa (COREDE, Serra) Gramado, Canela, Nova Petrópolis (COREDE, Hortências), Torres, Capão da Canoa e Arroio do Sal (COREDE, Litoral). 
Clínicas Odontológicas na pandemia; análise de dados; e considerações finais. Na próxima seção, será apresentado um breve histórico do COVID-19.

\section{COVID-19 e clínicas odontológicas}

Desde que a COVID-19 foi anunciada ao mundo, em 30 de janeiro de 2020, pela Organização Mundial da Saúde - OMS (2019), fez com que a comunidade científica se voltasse mais uma vez para o campo da saúde global. Deste modo, a pandemia SARS-CoV2 (Covid-19) chegou também ao Brasil, gerando um caos no ano de 2020, causando um impacto social, econômico e psicológico (BEZERRA et al., 2020; DE OLIVEIRA; CORRÊA, 2020; GULLO, 2020; ORNELL et al., 2020; NETO, 2020). O Coronavírus tornou-se a quinta pandemia documentada desde a pandemia de gripe espanhola de 1918 (MENG; HUA; BIAN, 2020), tendo sido registrada pela primeira vez em Wuhan, na China e, posteriormente, disseminada ao redor do mundo (LIU; KUO; SHIH, 2020). A pandemia COVID-19 acarretou a instauração de uma situação caótica, de modo que obrigou a sociedade como um todo a alterar suas práticas cotidianas. Diante de tal cenário, os diversos setores da sociedade foram atingidos, seja do ponto de vista econômico ou da qualidade de vida. As pessoas tiveram que ressignificar suas práticas de consumo (BEZERRA et al., 2020; MENG; HUA; BIAN, 2020; SENHORAS, 2020).

A sustentação econômica do sistema financeiro, a saúde mental das pessoas em tempos de confinamento, e o temor pelo risco de adoecimento e morte, acesso aos bens essenciais como alimentação, medicamentos, transporte, entre outros, também impactaram na vida das pessoas (FIOCRUZ, 2020; GHANI, 2020). A pesquisa científica na área da medicina, que explora as especificidades sobre a COVID-19, está em pleno andamento em nível mundial (CARRER et al., 2020; DA SILVA et al., 2020; MENG et al., 2020). Diuturnamente, os cientistas têm se dedicado ao desenvolvimento e a testes com vacinas e medicamentos para dirimir os altos índices de contágio do COVID-19 (DA SILVA et al., 2020; FERGUSON et al., 2020; FIOCRUZ, 2020). Além de que, a necessidade de ações para contenção da mobilidade social como isolamento e quarentena, bem como a velocidade e urgência de testagem de medicamentos e vacinas evidenciam implicações éticas e de direitos humanos que merecem análise crítica e prudência (FIOCRUZ, 2020; PAHO, 2020;).

A Organização Mundial de Saúde - OMS (2020), acerca da transmissibilidade do novo vírus, sugere como estratégia na maioria dos países, a continuidade do distanciamento social e a frequente higienização das mãos. Nesse sentido, a primeira reação dos países, diante do 
surto do COVID-19, foi de fechar instituições de ensino, suspender reuniões sociais, atividades esportivas, eventos, aeroportos e até bancos na tentativa de controlar a propagação do vírus. Além disso, vários indivíduos entraram em autoquarentena para fazer sua parte na sociedade, de modo a, possivelmente, dirimir a propagação do vírus, em especial, quando associado a comorbidades (OMS, 2020).

No entanto, as entidades de saúde são de extrema relevância para a sociedade e não podem deixar de atender à comunidade, dificilmente podem fechadas durante uma pandemia, salvo quando existe um surto dentro dessas entidades de saúde (ARTESE, 2020; SCHWENDICKE; KROIS; GOMEZ, 2020). Dentre os profissionais da saúde estão os cirurgiões-dentistas. Apesar destes profissionais terem um alto padrão de conhecimento e prática em uso de equipamento de proteção individual (EPIs), os cirurgiões-dentistas, assim como os demais profissionais da saúde, se encontram em um estado de ansiedade e medo (ATHER et al., 2020; MENG; HUA; BIAN, 2020). Várias clínicas odontológicas modificaram seus serviços de acordo com as diretrizes recomendadas. Estas voltaram-se apenas para tratamento de emergência, levando a suspensão de consultas eletivas nos consultórios por um período incerto (GHANI, 2020; MENG; HUA; BIAN, 2020).

Os cirurgiões-dentistas, assim como toda a população, estão cada dia mais apreensivos diante da nova realidade, uma vez que a propagação do COVID-19 vem aumentando (FERGUSON et al., 2020). Diante deste cenário, além do medo de contrair e transmitir a COVID-19, há a incerteza sobre o futuro econômico do país (ARTESE, 2020; SILVA et al., 2020). Países como o Brasil, que estava emergindo de uma crise econômica em 2020, terão que arcar com uma significativa recessão (BANCO CENTRAL DO BRASIL, 2020). De acordo com Schwendicke, Krois e Gomez (2020) quando realizaram uma pesquisa na Alemanha composta por amostragem aleatória de 300 dentistas, concluíram que o COVID-19 já apresentava um impacto econômico negativo nas práticas odontológicas em 2020 (SCHWENDICKE; KROIS; GOMEZ, 2020). Por sua vez, na Itália, a atividade odontológica foi reconhecida como um serviço de primeira necessidade pelo Decreto Ministerial $\mathrm{n}^{\circ} 19$ de 22 de março de 2020 (ITÁLIA, 2020) determinou que durante a pandemia de COVID-19, a prática odontológica fosse limitada aos tratamentos de urgência e emergência, enquanto tratamentos eletivos fossem adiados. 


\section{Metodologia}

Este é um estudo de caráter exploratório descritivo realizado por meio de um questionário do tipo survey (MALHOTRA, 2006), enquanto pesquisa quantitativa, composta por dados amostrais coletados por meio de questionário estruturado. $\mathrm{O}$ referido instrumento visa a analisar uma amostra da população e destina-se a obter informações específicas dos entrevistados (MALHOTRA, 2006).

A abordagem voltada às clínicas e aos consultórios no período de pandemia é parte constitutiva de uma pesquisa de doutoramento na área da Saúde, relacionada à Odontologia e aprovada pelo Comitê de Ética em Pesquisa (CEP) da Universidade em que esta pesquisa está inscrita (revisão às cegas), com parecer de número CAAE: 39814720.9.0000.5341. No que tange à coleta de dados referente às práticas das clínicas odontológicas, essa deu-se por meio de um questionário semiestruturado, composto por 27 perguntas, a saber, 16 questões abertas e 11 questões fechadas, dividida em 03 eixos: eixo 01 - dados identificação de clínica; eixo 02 - funcionamento da clínica; e eixo 03 - gestão econômica. A partir dos eixos norteadores citados, averiguou-se quais foram os impactos econômicos nas clínicas odontológicas. Diante do exposto, a pesquisa contou com a participação de 126 sócios-proprietários de clínicas odontológicas, que responderam ao instrumento de pesquisa. Não foi necessário nenhum tipo de identificação dos respondentes e a participação foi voluntária. A aplicação do questionário ocorreu no período de 06 de janeiro a 31 de maio de 2021, de modo presencial quando ocorreu a entrega e a coleta dos questionários.

O cálculo amostral foi feito a partir do software Statulator ${ }^{5}$. Quanto ao fator de interesse, tendo como tamanho populacional 220 respondentes, o estudo exigia um tamanho mínimo amostral de 140 respondentes. Em outras palavras, inferiu-se que, a partir de 140 respondentes, $50 \%$ destes tinham fator de interesse, o que representou $95 \%$ de fator confiança. No entanto, foram contabilizados apenas 126 questionários, uma vez que 14 questionários retornaram em branco.

Para a análise de dados utilizou-se o pacote da Microsoft planilhas do Excel, para fins de análise e tabulação dos dados coletados. Além disso, os dados lapidados foram organizados em gráficos. A análise de dados teve o aporte teórico-metodológico na área de sustentabilidade econômica (DE OLIVEIRA et al., 2020; GULLO, 2020; MILLER, 2019; NETO, 2020), da gestão econômica (FERRAZ, 2020; SCHWENDICKE; KROIS; GOMEZ, 2020) pandemia e da Odontologia (HADDAD et al., 2014). A partir do referencial teórico

${ }^{5}$ Disponível em: http://statulator.com/SampleSize/ss1P.html. Acesso em: 10 dez. 2020.

Temas em Educ. e Saúde, Araraquara, v. 17, n. 00, e021015, 2021 
citado, investigavam-se aspectos como: (a) houve o aumento ou a redução de gastos com recursos materiais e humanos? (b) houve necessidade de redução de carga horária de trabalho, houve redução de receita no período da pandemia no ano de 2020?; (c) a clínica possui planejamento financeiro?; (d) a clínica tem ou fez reserva financeira para períodos de crise?; (e) se sim, por quanto tempo a clínica conseguiria se manter?. Houve, deste modo, desenvolvimento de possíveis estratégias institucionais para a oferta de atendimentos online, agendamentos e orientações?

\section{Análise de dados}

Diante dos dados analisados, a partir dos 03 eixos propostos nesta pesquisa, o primeiro aspecto evidenciado, quando realizada a análise do eixo 2 deste estudo foi a necessidade de redução na carga horária dos funcionários e da carga horária de atendimento. Dos 126 respondentes, $61,1 \%$ responderam que tiveram redução de carga horária no atendimento e $38,9 \%$ responderam que não houve redução na carga horária.

De acordo com pesquisas realizadas na área da Odontologia (GHANI, 2020; LUCENA et al., 2020) em outras regiões, essa redução no atendimento das clínicas odontológicas ocorre(u) porque houve/há uma forte recomendação de que a população respeitasse as orientações acerca do isolamento social. Neste horizonte, alguns indivíduos mostram-se preocupados em sair de casa para procurar os serviços na área da saúde, o que reverberou também quanto aos serviços oferecidos pelas clínicas odontológicas, de modo que isso, resultou na redução do número de atendimentos e, consequentemente, ocasionou uma busca por atendimento apenas em casos extremos

Somado a isso, ainda se tem a falta de clareza da população, quanto à compreensão do que, de fato, seria uma situação de urgência ou emergência odontológica. Nestes casos, o fator dor pareceu ser determinante para que o paciente busca-se atendimento odontológico (GHANI, 2020; LUCENA et al., 2020). De acordo com Medeiros (2020), uma pesquisa realizada na China, revelou queda de $38 \%$ no número de pacientes atendidos em serviços de urgências odontológicas durante o período pandêmico.

O segundo aspecto revelado pela análise de dados, refere-se à redução de receita durante a pandemia. Quanto à redução de receita, 68,3\% dos respondentes mencionaram que houve redução; 19\% que não houve redução e 12,7\% responderam que se mantiveram estáveis economicamente, como nos períodos anteriores à pandemia. Cabe salientar, a partir das afirmações de Carrer et al. (2020) e Gomes et al. (2021), que o predomínio da redução de 
receita ocorreu não somente nas regiões Serra, Hortênsias e Litoral do RS. Isso porque, o mesmo fato vem ocorrendo de forma global. Ainda, segundo Carrer et al, (2020), a crise na saúde pública resultante do COVID-19, tem apresentado globalmente impactos negativos na atividade odontológica.

Segundo Lucena et al. (2020), os atendimentos de casos de inflamação e dor de dente no Brasil caíram cerca de 30\%, quando comparados com o mesmo período no ano de 2019 . Referente aos casos de primeira consulta odontológica, notou-se redução de cerca de $40 \%$, haja vista os pacientes estarem evitando sair de suas residências. Tal redução teve por base os dados coletados por meio da página do Sistema Único de Saúde Atenção Básica (e-SUSAB), no portal do Ministério da Saúde, que permite identificar o registro de baixo índice de consultas odontológicas durante a pandemia de COVID-19 (BRASIL, 2020).

Devido às características dos ambientes odontológicos, o risco de infecção cruzada pode ser alto entre pacientes e dentistas. Para consultórios odontológicos e hospitais em áreas que são (potencialmente) afetadas com COVID-19, protocolos de controle de infecção rigorosos e eficazes são, urgentemente, necessários. Nesse sentido, o terceiro aspecto identificado refere-se às despesas da clínica relativo aos materiais que mais foram consumidos pelas clínicas odontológicas no período pandêmico de 2020. A pesquisa apontou que as clínicas odontológicas tiveram um aumento de $72,8 \%$ quanto ao consumo de materiais relacionados aos Equipamentos de Proteção de Individual - EPIs durante o período pandêmico. São considerados (EPI) qualquer meio ou dispositivo destinado a ser utilizados por uma pessoa contra possíveis riscos ameaçadores da sua saúde ou segurança durante o exercício de uma determinada atividade (MILFONT; OLIVEIRA 2015). À medida que a epidemia acelera no Brasil, o acesso a equipamentos de proteção individual (EPI), tais como máscaras, luvas... para profissionais de saúde é uma preocupação constante.

Isto, pois de acordo com Meng, Hua e Bian (2020), as medidas de controle da infecção são necessárias para evitar que o coronavírus se espalhe ainda mais e para ajudar a controlar a situação epidêmica, neste caso, pandêmica. Diante disso, há um consenso em relação às orientações sobre a prestação de serviços odontológicos durante a pandemia do COVID-19, em que é preconizado que os dentistas reforcem as medidas de segurança através dos equipamentos de proteção individual (EPIs) e, evitem, quando possível, a realização de procedimentos que envolvam a produção de aerossóis e de gotículas (OLIVEIRA et al., 2020), a fim de prevenir controlar a propagação do vírus.

O quarto aspecto refere-se à dependência das clínicas quanto ao serviço terceirizado, a maior dependência é de laboratórios de próteses dentárias com 70,2\%, seguido do 
recolhimento de resíduo contaminado com $16,9 \%$ e, 12,9\% dos participantes, responderam que não dependem de serviços de terceiros. A pesquisa destaca a relevância e a dependência dos serviços de terceiros para o funcionamento de uma clínica odontológica. Destaca-se aqui a coleta de resíduo contaminado, dada a dimensão e a intensificação promovida pela COVID19, o que demandou uma readequação no agir desses prestadores de serviços. Com a pandemia, esse serviço tornou-se imprescindível. Entende-se que é preciso que estes profissionais façam o descarte correto do resíduo, assim contribuirão para que haja uma menor propagação do vírus por meio de material infectado. O quinto aspecto refere-se ao planejamento financeiro e às reservas financeiras das clínicas odontológicas. A pesquisa constatou que $52,4 \%$ dos respondentes têm planejamento financeiro ou uma reserva financeira para períodos de crise; 47,6 \% dos participantes não possuem esse recurso. Apesar das clínicas constituírem planejamento e reserva financeira, estas não são pensadas para longo prazo, conforme dados analisados. Ou seja, as reservas financeiras variam de 1 a 12 meses para este percentual, enquanto $45 \%$ dos entrevistados não souberam responder por quanto tempo conseguiria se manter com as reservas financeiras.

De acordo com Dornela et al. (2014) ter um planejamento financeiro saudável é: guardar uma parte de seus rendimentos, para formar um bom patrimônio; começar a poupar o quanto antes, para chegar à velhice sem depender de ninguém; sempre pensar no futuro, levando em conta o médio e o longo prazo; ter um propósito, fazer o planejamento das aplicações pensando nesses ideais. É essencial que no atual cenário econômico os profissionais liberais, e especialmente os odontólogos, devam estar preparados com estratégias e ferramentas que auxiliem na gestão, principalmente a financeira (GOMES; MORAES, 2013).

O sexto aspecto refere-se no "onde" se concentra o maior custo das clínicas odontológicas. De acordo com o gráfico 1 (um), o maior custo se concentra na aquisição de materiais. De acordo com Medeiros (2020) isso ocorre, porque a maioria dos materiais utilizados nas clínicas odontologicas são importadas da China, o que nos leva a uma dependência do mercado estrangeiro. 


\section{Gráfico 1 - Custos}

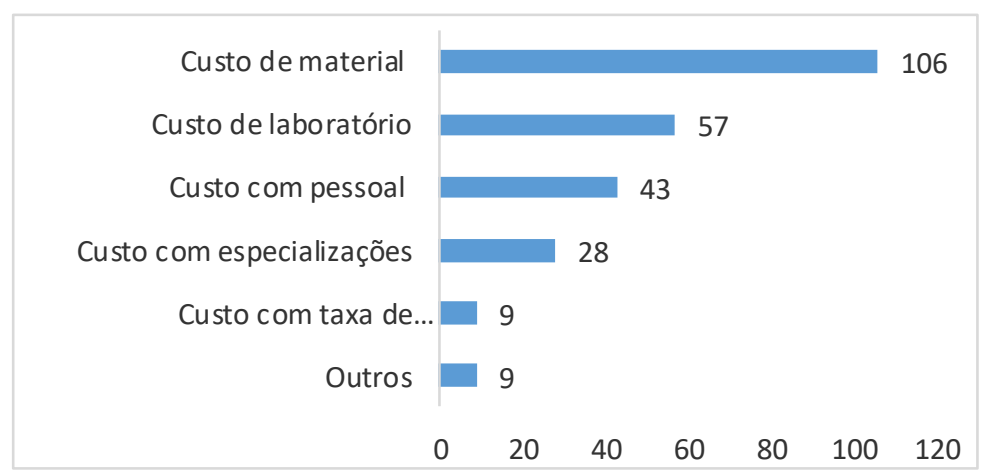

Fonte: Elaborado pelos autores

O sétimo aspecto refere-se ao atendimento online nas clínicas odontológicas. A pesquisa indicou que $86,5 \%$ dos respondentes não vêm viabilidade para o atendimento online e não fizeram uso dessa ferramenta durante o período pandêmico de 2020; 13,5\% responderam que sim que há possibilidade de dar orientações, indicações e avaliações por meio do atendimento online, e fizeram uso dessa ferramenta no ano de 2020. Contudo Carrer et al. (2020) afirmaram que esta ferramenta tem se mostrado eficaz tanto no custo quanto na disseminação do acesso, com vantagens relacionadas ao aumento da efetividade do serviço; da integralidade; e o acesso universal, com ganhos significativos de qualidade e precisão da imagem, à redução do tempo de espera e dos custos de tratamento. A utilização de Tecnologias Digitais de Informação e Comunicação (TDIC) proporciona uma percepção da sociedade atual, mostra a interligação global e promove o acesso contínuo e permanente a toda e qualquer informação (HADDAD et al., 2014; PACHECO et al., 2018). De acordo com Gullo (2020), o mundo se encaminha para um atendimento online, compreender como o seu negócio pode se beneficiar desse novo momento é uma questão de sobrevivência e vale para todos os segmentos da economia.

\section{Considerações finais}

Os dados analisados demonstraram que; houve redução carga horária dos funcionários, do atendimento, e consequentemente de receita. Esta redução é um reflexo das medidas de precaução que, foram anunciadas, durante o período pandêmico, visando à redução da transmissão da COVID-19 no de 2020.

Outro dado analisado está relacionado aos EPIs. À medida que a pandemia acelera no Brasil, o acesso aos Equipamentos de Proteção Individual (EPIs) para profissionais de saúde é 
uma preocupação constante. Pontualmente no início e durante este período pandêmico, o que vemos, é a alta dos preços dos EPIs, especialmente máscaras e aventais descartáveis. Estes equipamentos tiveram aumentos expressivos associados ao desabastecimento do mercado de insumos.

Em períodos pandêmicos, não basta apenas a fazer a gestão da clínica, ter o controle e organização do consumo e das compras dos insumos, é necessário acompanhar o mercado econômico no Brasil e no mundo, com atenção redobrada para garantir o abastecimento de insumos, medicamentos, equipamentos e mão de obra qualificada.

Atualmente, há uma grande oferta de serviços odontológicos, o que leva os profissionais cirurgiões-dentistas a oferecerem um serviço diferenciado para obter vantagem competitiva. Tal diferenciação não depende somente dos aspectos técnicos relacionados ao bom exercício da Odontologia, mas também no que diz respeito à gestão de um consultório odontológico, ou seja, um bom planejamento estratégico, uma análise financeira e administração de pessoal contribuem para um bom desempenho financeiro. Os impactos da pandemia da COVID-19 provocaram impactos econômicos negativos assimétricos, de natureza trans escalar e intertemporal, gerando efeitos na economia mundial que ressoam em distintos graus de sensibilidade e vulnerabilidade macroeconômica dos países e microeconômica das cadeias globais de produção e consumo (SENHORAS, 2020).

De acordo com o Fundo Monetário Internacional (FMI, 2020) a pandemia do COVID19 tem um impacto muito grande sobre a economia e a política internacional. Por mais que o risco de uma crise sanitária mundial, causada por um vírus vindo da Ásia exista a muitos anos, a grande maioria dos países demonstrou estar despreparada para suas consequências (GULLO, 2020). A pesquisa também apontou que a alternativa de atendimento online é uma ferramenta pouco explorada nas clínicas odontológicas abordadas. Apesar desta ferramenta ter sido amplamente utilizada durante o período pandêmico de 2020 em outras áreas.

Sendo assim, as clínicas odontológicas deveriam voltar-se para uma sustentabilidade econômica, buscando viabilidade financeira, retorno do investimento realizado, alocação e gerenciamento mais eficientes dos recursos disponibilizados no mercado de trabalho, procurando um contínuo aprimoramento, na ciência e tecnologia.

AGRADECIMENTOS: O presente trabalho foi realizado com apoio da Coordenação de Aperfeiçoamento de Pessoal de Nível Superior - Brasil (CAPES) - Código de Financiamento $001 "$. 


\section{REFERÊNCIAS}

ARTESE, F. Covid-19: The aftermath for orthodontics. Dental Press Journal of Orthodontics, v. 25, n. 2, p. 7-8, 2020. DOI: https://doi.org/10.1590/2177-6709.25.2.007008.edt

ATHER, A. et al. Coronavirus disease 19 (COVID-19): implications for clinical dental care. Journal of Endodontics, v. 46, n. 5, p. 584-595, 2020. DOI:

https://doi.org/10.1016/j.joen.2020.03.008

BEZERRA, A. C. V. et al. Fatores associados ao comportamento da população durante o isolamento social na pandemia de COVID-19. Ciência \& Saúde Coletiva, v. 25, p. 24112421, 2020. DOI: https://doi.org/10.1590/1413-81232020256.1.10792020

BRASIL. Banco Central do Brasil. Comitê de Comércio e Serviços Enfrentamento dos Efeitos Econômicos da COVID-19. 15, jun. 2020. Disponível em:

https://www.bcb.gov.br/conteudo/homeptbr/TextosApresentacoes/Ap_RCN_CCS_29.6.2020.pdf. Acesso em: 10 abr. 2021.

CAGETTI, M. G. et al. COVID-19 outbreak in North Italy: an overview on dentistry. A questionnaire survey. International journal of environmental research and public health, v. 17, n. 11, p. 3835, 2020. DOI: https://doi.org/10.3390/ijerph17113835

CARRER, F. C. A. et al. A COVID-19 na América Latina e suas repercussões para a odontologia. Revista Panamericana de Salud Pública, v. 44, p. e66, 2020.DOI: 10.26633/RPSP.2020.66

CHEN, J. Pathogenicity and transmissibility of 2019-nCoV - a quick overview and comparison with other emerging viruses. Microbes and Infection, v. 22, n. 2, p. 69-71, 2020. DOI: https://doi.org/10.1016/j.micinf.2020.01.004

DA SILVA, R. O. C. et al. Protocolos de atendimento odontológico durante a pandemia de COVID-19 nos países do MERCOSUL: similaridades e discrepâncias. Vigilância Sanitária em Debate: Sociedade, Ciência \& Tecnologia, v. 8, n. 3, p. 86-93, 2020. DOI: https://doi.org/10.22239/2317-269x.01620

DE OLIVEIRA, J. J. M. et al. O impacto do coronavírus (covid-19) na prática odontológica: desafios e métodos de prevenção. Revista Eletrônica Acervo Saúde, n. 46, p. e3487, 2020. DOI: https://doi.org/10.25248/reas.e3487.2020

DE OLIVEIRA, R. M.; CORRÊA, Y. Ensino de língua portuguesa com a mediação das tecnologias digitais em tempos de pandemia. Dialogia, n. 36, p. 252-268, 2020.

DORNELA, F. J.et al. Educação Financeira: aprendendo a lidar com dinheiro. Raízes e Rumos, v. 2, n. 1, 2014.

FERGUSON, N. et al. Report 9: Impact of non-pharmaceutical interventions (NPIs) to reduce COVID19 mortality and healthcare demand. Imperial College, 2020. DOI:

https://doi.org/10.25561/77482 
FERRAZ, A. R. As grandes Pandemias da História. Revista de Ciência Elementar, v. 8, n. 2, 2020. DOI: https://doi.org/10.24927/rce2020.025

FIOCRUZ. Fundação Oswaldo Cruz. Impactos sociais, econômicos, culturais e políticos da pandemia. 2020. Disponível em: https://portal.fiocruz.br/impactos-sociais-economicosculturais-e-politicos-da-pandemia. Acesso em: 10 abr. 2020.

FMI. Fundo Monetário Internacional. A resposta do FMI ao COVID-19. 2021. Disponível em: https://www.imf.org/en/About/FAQ/imf-response-to-covid-19. Acesso em: 10 abr. 2021

GHANI, F. Covid-19 Outbreak-Immediate and long-term impacts on the dental profession. Pakistan Journal of Medical Sciences, v. 36, n. COVID19-S4, p. S126, 2020.DOI: https://doi.org/10.12669/pjms.36.COVID19-S4.2698

GOMES, M. J. O.; MORAES, L. S. A Importância do fluxo de caixa para a organização financeira da Empresa X. Revista Científica Semana Acadêmica, Fortaleza, ano MMXI, n. 000006, 2013.

GOMES, P. et al. O Impacto do Coronavírus (COVID-19) as atividades odontológicas: desafios econômicos e mentais. Research, Society and Development, v. 10, n. 1, e22310111207.DOI: https://doi.org/10.33448/rsd-v10i1.11207

GULLO, M. C. A economia na pandemia Covid-19: algumas considerações. ROSA DOS VENTOS-Turismo e Hospitalidade, v. 12, n. 3, 2020. DOI:

http://dx.doi.org/10.18226/21789061.v12i3a05

HADDAD, A. E. et al. Research in the field of health, dentistry, telehealth and teledentistry. Brazilian Oral Research, v. 28, n. 1, p. 1-2, 2014.DOI: https://doi.org/10.1590/18073107bor-2014.vol28.0001

HAIR, J. F. et al. Análise multivariada de dados. Bookman editora, 2009.

ITÁLIA. Decreto del Presidente del Consiglio dei Ministri 11 Marzo 2020. Gazzetta Ufficiale Della Repubblica Italiana, 2020. Disponível em: https://www.gazzettau_ciale.it/eli/id/2020/03/11/20A01605/sg. Acesso em: 10 mar.2021.

LIU, Y.-C.; KUO, R.-L.; SHIH, S.-R. COVID-19: The first documented coronavirus pandemic in history. Biomedical Journal, v. 43, n. 4, p. 328-333, 2020.DOI: https://doi.org/10.1016/j.bj.2020.04.007

LUCENA, E. H. G. et al. Acesso em saúde bucal na atenção básica antes e após o início da pandemia de COVID-19 no Brasil. Pesquisa Brasileira em Odontopediatria e Clínica Integrada, 2020. DOI: https://doi.org/10.1590/SciELOPreprints.819

MALHOTRA, N. K. Pesquisa de marketing: uma orientação aplicada. Bookman, 2006.

MEDEIROS, E. A. S. Health professionals fi ght against COVID-19. Acta Paulista de Enfermagem, v. 33, n. 3, p. I-IV, 2020. DOI:https://doi.org/http://dx.doi.org/10.37689/actaape/2020EDT0003 
MENG, L.; HUA, F.; BIAN, Z. Coronavirus disease 2019 (COVID-19): emerging and future challenges for dental and oral medicine. Journal of Dental Research, v. 99, n. 5, p. 481-487, 2020. DOI: https://doi.org/10.1177/0022034520914246

MILFONT, J. A. C.; OLIVEIRA, A. H. A. Equipamentos de proteção individual em odontologia: revisão integrativa de literatura. INTERFACES, v. 3, n. 8, p. 1-6, 2015. DOI: https://doi.org/10.16891/2317-434X.268.ISSN

\section{MILLER, A. R. Perspectivas no gerenciamento da Covid-19 no atendimento} odontológico. 2021. Dissertação (Mestrado Profissional em Administração - Gestão em Sistemas de Saúde) - Universidade Nove de Julho, São Paulo, SP, 2021.

NETO, R. B. G. Impactos da covid-19 sobre a economia mundial. Boletim de Conjuntura (BOCA), v. 2, n. 5, p. 113-127, 2020. DOI: http://dx.doi.org/10.5281/zenodo.3786698

ORNELL, F. et al. Pandemia de medo e Covid-19: impacto na saúde mental e possíveis estratégias. Revista Debates in Psychiatry, p. 2-7, 2020.

PACHECO, K. T. S. et al. Tecnologias de informação e comunicação para formação profissional em odontologia: uma proposta do Telessaúde/ES. Rev. ABENO, p. 127-136, 2018. DOI: https://doi.org/10.30979/rev.abeno.v18i3.548

PAHO. Pan American Health Organization. Considerations for public health measures related to schools in the context of COVID-19. Annex to the Considerations for the Adjustment of Social and Public Health Measures in the Context of COVID-19, 2020. Disponível em: https://iris.paho.org/handle/10665.2/52682. Acesso em: 10 abr.2021

POTT, E. T. B.; POTT-JUNIOR, H. A percepção dos estudantes de medicina sobre sua participação em um programa de enfrentamento ao COVID-19. Temas em Educação e Saúde, v. 17, n. 00, p. e021001, 2021. DOI: 10.26673/tes.v17i00.14681

SCHWENDICKE, F.; KROIS, J.; GOMEZ, J. Impact of SARS-CoV2 (Covid-19) on dental practices: Economic analysis. Journal of Dentistry, v. 99, p. 103387, 2020.DOI: https://doi.org/10.1016/j.jdent.2020.103387

SENHORAS, E. M. COVID-19: enfoques preventivos. EdUFRR, 2020.

WHO. World Health Organization. Clinical management of severe acute respiratory infection when Middle East respiratory syndrome coronavirus (MERS-CoV) infection is suspected: interim guidance, 2019. Disponível em: https://apps.who.int/iris/handle/10665/178529. Acesso em: maio.2021 


\section{Como referenciar este artigo}

BRINGMANN, D. R.; GULLO, M. C. R.; GAMBA, T. O. O impacto da COVID-19 na sustentabilidade econômica das clínicas odontológicas que fazem parte do COREDEs Serra, Hortências e Litoral do RS. Temas em Educ. e Saúde, Araraquara, v. 17, n. 00, e021015, 2021. e-ISSN 2526-3471. DOI: https://doi.org/10.26673/tes.v17i00.15260

Submetido em: 30/06/2021

Revisões requeridas: 11/07/2021

Aceito em: 21/07/2021

Publicado em: 19/08/2021 